\title{
Time-varying Response Analysis of Spur Gear System
}

\author{
Chao $\mathrm{Li}^{1, *}$, Hongwei Liu ${ }^{1}$ \\ ${ }^{1}$ Department of Computer, Jiangsu Automation Research Institute, Lianyungang, China
}

\begin{abstract}
In this paper, a space-driven two-stage spur gear system is taken as the research object, and a 10 DOF dynamic model is established. Considering the high load characteristics of the space drive system and the time-varying stiffness and tooth clearance of the gear system, a nonlinear dynamic response analysis was performed. The characteristics of the vibration acceleration, shock and transmission error of the gear system are studied in this paper. This paper analyzes the relationship between backlash and return difference, and derives the theoretical formula between the two. The time-varying stiffness was corrected to make the theoretical model closer to reality. The research in this paper enriches the study on space drive systems and high load gear systems.
\end{abstract}

\section{Introduction}

Reference [1] takes a typical two-stage spur gear system of a wind turbine as the research object, establishes a nonlinear dynamic equation, and gives the excitation to obtain the dynamic response of the shaft, bearing and contact teeth. The model in [2] includes load conditions that change with time, and considering the non-linearity of the contact between the teeth, the nonlinear response of the gear under different loads is studied. Reference [3] obtained the grid force by a combination of numerical and analytical methods, and successfully applied it to the simulation of sample transmission vibration. Reference [2] established a dynamic model of a spur gear pair, including backlash, time-varying stiffness, and static transmission error. The effects of damping ratio and excitation amplitude on the frequency response curve were studied. Reference [4] verified that backlash is the main source of positioning error and impact through dynamic response analysis. Reference [5] applied a multi-scale method to analyze the nonlinear torsional instability of the flexible shaft gear system. Reference [2] analyzed the influence of gear dynamic changes on backlash changes, and established an indirect relationship between gear dynamic performance and tooth surface characteristics.

The analysis of the dynamic characteristics of the large inertia load space drive mechanism is less relevant in the theoretical research, which mainly utilizes simulation technology for both analysis and research. Tianfu Yang considered the impact of a high reduction ratio and studied the dynamic characteristics of the space manipulator, whereas the characteristics of the large inertia load were not analyzed. Zhigang $\mathrm{Xu}$ proposed an equivalent simulation method for an excessively large inertia load, simulating the large moment of inertia required by the space manipulator and verified the effectiveness of the method by numerical simulation under different working conditions. Chen Shiqiestablished a bending - torsion coupling nonlinear dynamic model of a low speed overload planetary gear drive system with friction and studied the effect of friction on the nonlinear dynamic behavior of a low speed overload gear transmission. Ahmed Hammami developed a model of planetary gear torsional concentrated parameter with dynamic recirculation and studied the nonlinear behavior under variable load conditions.

The effects of error incentives on the system and the relationship between the gear machining errors and the system dynamic characteristics can provide guidance for the determination of the accuracy grade in the gear design and the selection of the machining method. Fernández added profile and pitch errors in the dynamic model, executing tooth profile modification and validated the validity of the model through the examples simulation under different torques. A gear dynamics test set-up with integrated root strain and dynamic transmission error measurement systems was described by M.A. Hotait, in which dynamic factor and dynamic transmission error measurements from both unmodified and modified spur gears were presented and the corresponding relationship was demonstrated experimentally. Tengjiao Lin utilized the finite element modeling to obtain the machining and assembly errors of the gear system, which was applied to the kinetic model and the dynamic transmission error was calculated. Guangjian Wang proposed the theoretical calculation equation of the no-load transmission error and studied the load variation frequency effect on the transmission error curve.

In this paper, the gear system of a high-load space drive mechanism is taken as the research object, and a 10degree-of-freedom nonlinear dynamic model is established in consideration of factors such as backlash and time-varying stiffness. Backlash and return difference are often not distinguished in previous studies, and some studies even conflate the two. This will lead to inaccurate

\footnotetext{
* Corresponding author: sansking @163.com
} 
modeling, the data obtained by the solution cannot be close to reality, and even wrong conclusions are drawn. This paper analyzes the relationship between return difference and backlash. The calculation formula between return difference and backlash was derived, which will provide theoretical support for subsequent research.
When solving the dynamic response of a two-stage gear transmission system, it is assumed that the radial stiffness and damping of the four bearings at the support bearings at both ends of the gear are equal. The torsional stiffness can be calculated from geometric dimensions and related material properties. When the tooth shape and pitch error of the system are not considered, the dynamic equation of the system becomes as shown in the following equation:

\section{Dynamics model two-stage gear system}

$$
\begin{aligned}
& m_{1} \ddot{y}_{1}=-k_{y 1} y_{1}-c_{y 1} \dot{y}_{1}+k_{t 1}\left(r_{b 1} \theta_{1}-r_{b 2} \theta_{2}-y_{1}+y_{2}\right) \\
&+ c_{t 1}\left(r_{b 1} \dot{\theta}_{1}-r_{b 2} \dot{\theta}_{2}-\dot{y}_{1}+\dot{y}_{2}\right) \\
& m_{2} \ddot{y}_{2}=-k_{y 2} y_{2}-c_{y 2} \dot{y}_{2}-k_{t 1}\left(r_{b 1} \theta_{1}-r_{b 2} \theta_{2}-y_{1}+y_{2}\right) \\
&-c_{t 1}\left(r_{b 1} \dot{\theta}_{1}-r_{b 2} \dot{\theta}_{2}-\dot{y}_{1}+\dot{y}_{2}\right) \\
& m_{3} \ddot{y}_{3}=-k_{y 3} y_{3}-c_{y 3} \dot{y}_{3}+k_{t 2}\left(r_{b 3} \theta_{3}-r_{b 4} \theta_{4}-y_{3}+y_{4}\right) \\
& \quad+c_{t 2}\left(r_{b 3} \dot{\theta}_{3}-r_{b 4} \dot{\theta}_{4}-\dot{y}_{3}+\dot{y}_{4}\right) \\
& m_{4} \ddot{y}_{4}=-k_{y 4} y_{4}-c_{y 4} \dot{y}_{4}-k_{t 2}\left(r_{b 3} \theta_{3}-r_{b 4} \theta_{4}-y_{3}+y_{4}\right) \\
& \quad-c_{t 2}\left(r_{b 3} \dot{\theta}_{3}-r_{b 4} \dot{\theta}_{4}-\dot{y}_{3}+\dot{y}_{4}\right) \\
& J_{1} \ddot{\theta}_{1}=k_{1}\left(\theta_{0}-\theta_{1}\right)+c_{1}\left(\dot{\theta}_{0}-\dot{\theta}_{1}\right)-r_{b 1}\left[k_{t 1}\left(r_{b 1} \theta_{1}-r_{b 2} \theta_{2}-y_{1}+y_{2}\right)\right. \\
&\left.\quad+c_{t 1}\left(r_{b 1} \dot{\theta}_{1}-r_{b 2} \dot{\theta}_{2}-\dot{y}_{1}+\dot{y}_{2}\right)\right] \\
& J_{2} \ddot{\theta}_{2}=-k_{2}\left(\theta_{2}-\theta_{3}\right)-c_{2}\left(\dot{\theta}_{2}-\dot{\theta}_{3}\right)+r_{b 2}\left[k_{t 1}\left(r_{b 1} \theta_{1}-r_{b 2} \theta_{2}-y_{1}+y_{2}\right)\right. \\
&\left.+c_{t 1}\left(r_{b 1} \dot{\theta}_{1}-r_{b 2} \dot{\theta}_{2}-\dot{y}_{1}+\dot{y}_{2}\right)\right] \\
& J_{3} \ddot{\theta}_{3}=k_{2}\left(\theta_{2}-\theta_{3}\right)+c_{2}\left(\dot{\theta}_{2}-\dot{\theta}_{3}\right)-r_{b 3}\left[k_{t 2}\left(r_{b 3} \theta_{3}-r_{b 4} \theta_{4}-y_{3}+y_{4}\right)\right. \\
&\left.+c_{t 2}\left(r_{b 3} \dot{\theta}_{3}-r_{b 4} \dot{\theta}_{4}-\dot{y}_{3}+\dot{y}_{4}\right)\right] \\
& J_{4} \ddot{\theta}_{4}=-k_{3}\left(\theta_{4}-\theta_{5}\right)-c_{3}\left(\dot{\theta}_{4}-\dot{\theta}_{5}\right)+r_{b 4}\left[k_{t 2}\left(r_{b 3} \theta_{3}-r_{b 4} \theta_{4}-y_{3}+y_{4}\right)\right. \\
&\left.+c_{t 2}\left(r_{b 3} \dot{\theta}_{3}-r_{b 4} \dot{\theta}_{4}-\dot{y}_{3}+\dot{y}_{4}\right)\right]
\end{aligned}
$$

\section{Analysis of time-varying response of gear system}

After all parameters in the above dynamic differential equation are determined, the ODE15s function in MATLAB is used to solve the numerical solution of the dynamic differential equation. Find the second derivative of the displacement in the direction $y$, and the value obtained is the vibration acceleration of the gear in the direction $^{a}$. Figure 1 shows the acceleration of the four gears in the direction $y$ when the transmission error is not considered. It can be seen that the vibration signals of the four gears are vibration signals with periodic shocks. 

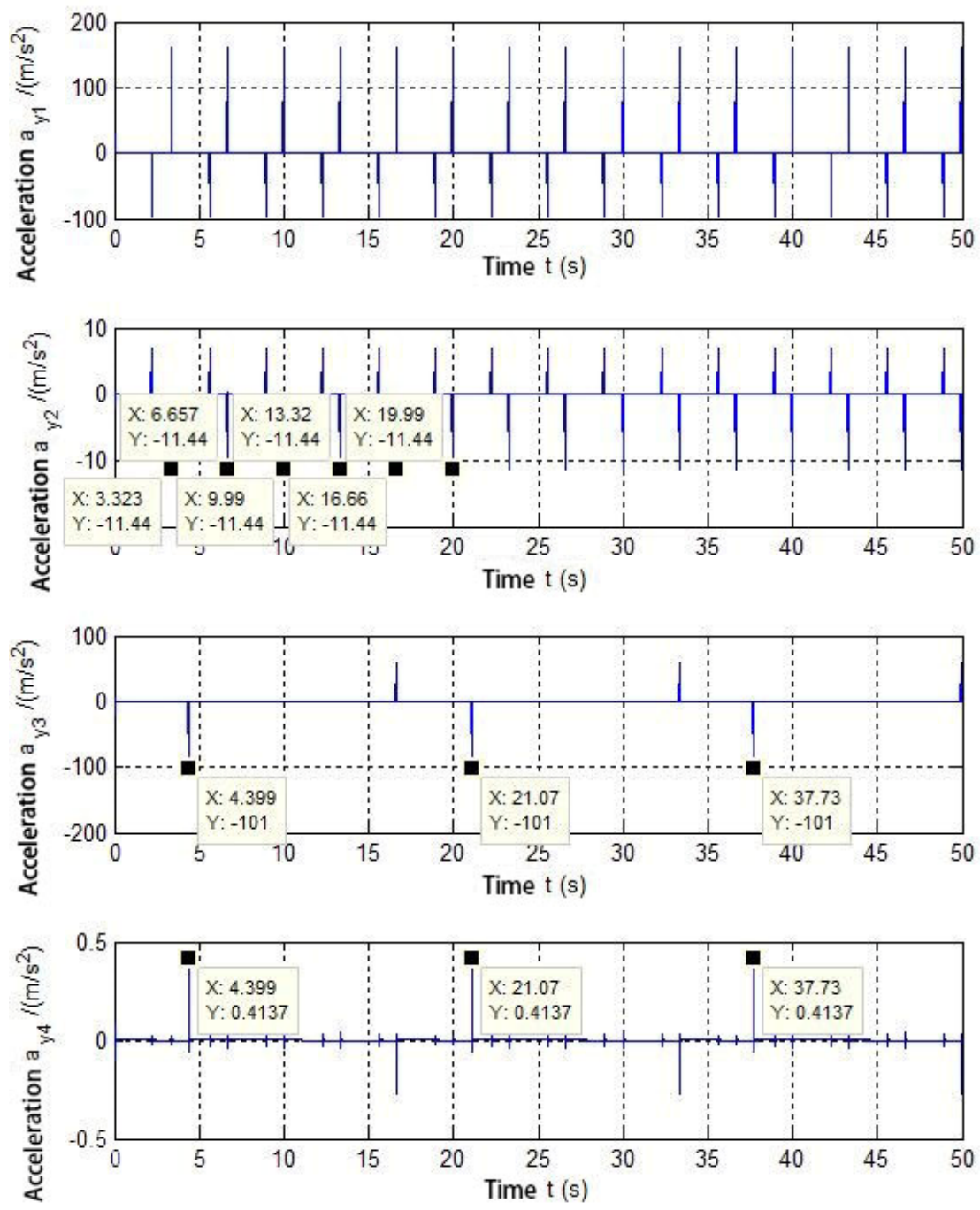

Figure 1 Curve of the vibration acceleration of gears

Figure 1 shows the response curve of the radial vibration acceleration of the gears during the time-varying stiffness of the four gears. This is mainly due to the stiffness impact caused by the change in the number of gear teeth that mesh simultaneously during the meshing process. Taking the acceleration response curve of the second gear in the figure as an example, the second gear in the figure is a response diagram of the acceleration of the large gear driven by the first gear in the y direction with time. It can be seen in the figure that the acceleration curve has shocks in two directions, the acceleration shocks in these two directions are caused by the time-varying meshing caused by the change in the number of gear pairs of gear 1 and gear 2 of the first stage gear The impact is generated, and the impact period of any two adjacent vibration acceleration times in the upward or downward direction is about left and right, which is determined by the angular velocity of the gear and the tooth angle of the gear 1.

The acceleration response curve of the gear 4 in the figure 1 above is locally enlarged, and the upward or downward impact point is generated. The response curve of the impact point is enlarged as shown in the figure below. It can be seen from the figure 2 that after the gear has an impact in the radial direction, it takes a period of time for the gear to stabilize again. This radial acceleration will cause radial displacement of the gear, cause changes in the position between gears, and even change the center distance of the gear, The change of the center distance will change the meshing accuracy of the gear transmission, which will also have a great interference on the smoothness of the gear transmission. 


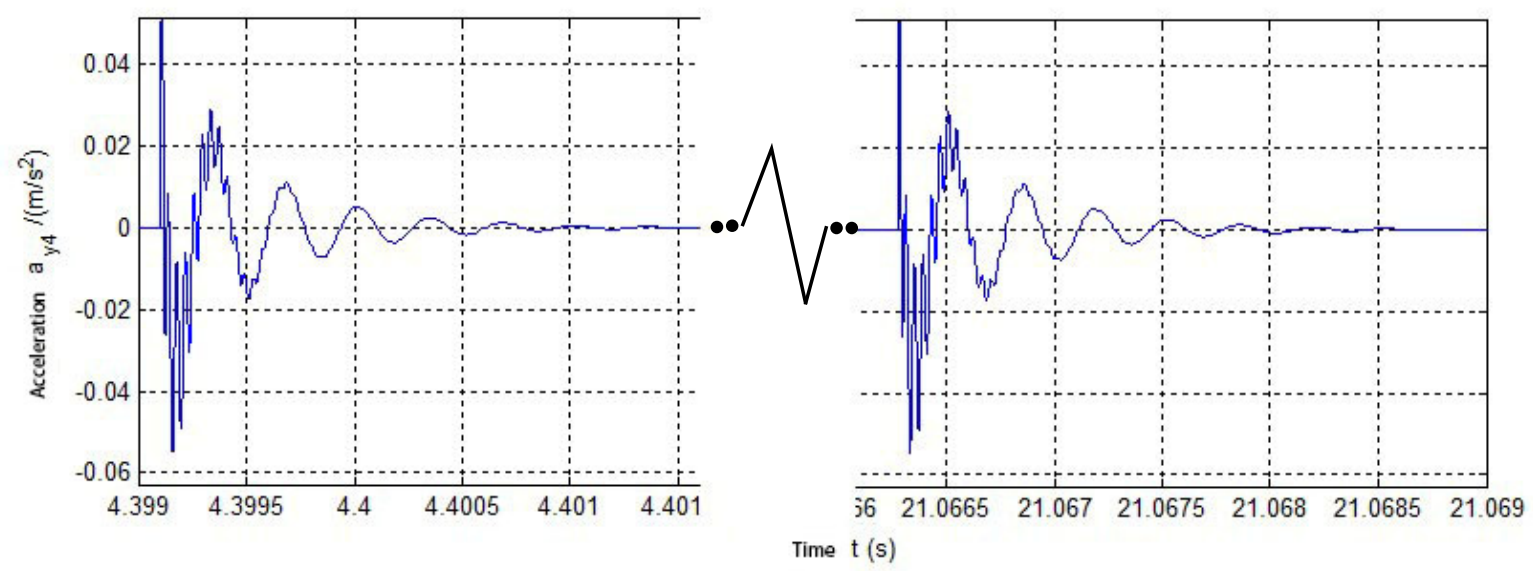

Figure 2 Partially enlarged graph of the impact response curve

The angular velocity impact amplitude of the gear 4 is $0.001379688 \mathrm{rad} / \mathrm{s}$ irrespective of the energy loss. The theoretical average output angular velocity of gear 4 should be $0.001047 \mathrm{rad} / \mathrm{s}$, which has exceeded the theoretical output average. This is very unfavorable to the system, and will cause motion accuracy and smoothness at the load end of the system.

At this time, the transmission error curve of the gear system is shown in the figure below. It can be seen from the figure that the transmission error of the system exhibits two periodic changes. And in each long cycle of $16.664 \mathrm{~s}$, it can be clearly seen that there are 5 short cycles with a time of $3.33 \mathrm{~s}$, as shown in the interval $\mathrm{A} \sim \mathrm{E}$ in the figure.

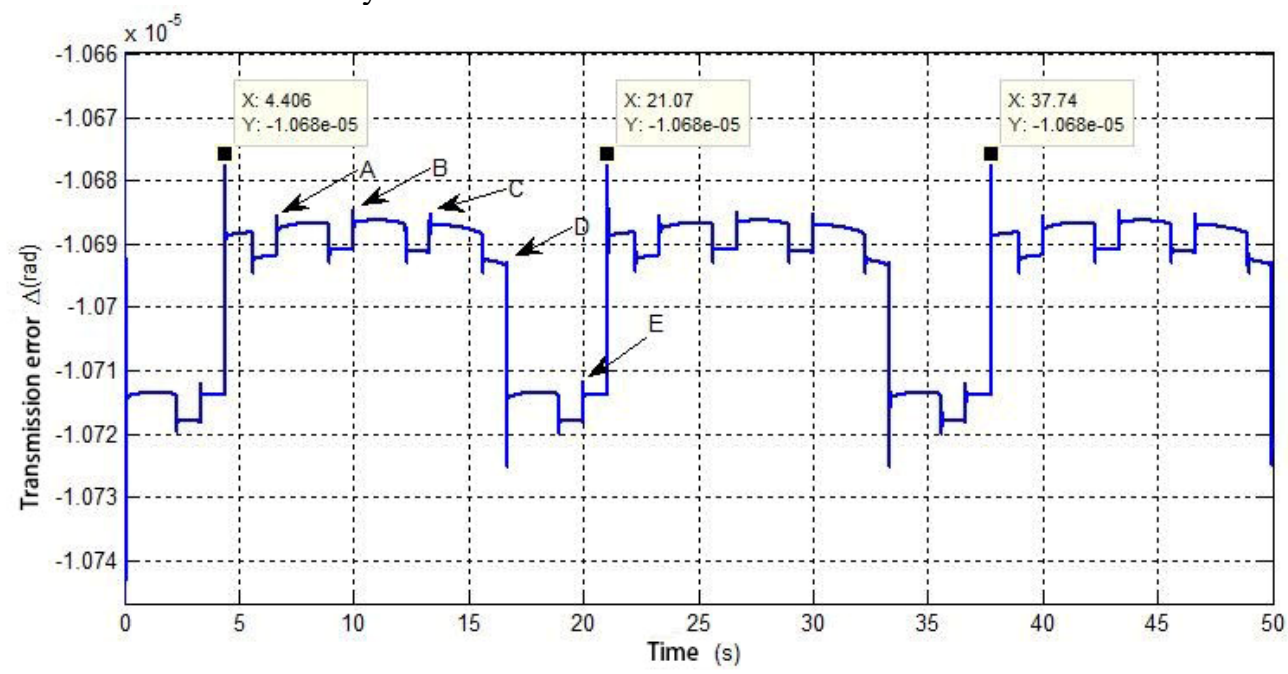

Figure 3 Transmission error curve

\section{Conclusion}

Taking the two-stage spur gear space-driven deceleration system as the research object, a 10-DOF nonlinear timevarying dynamic model was established. The ODE solver was used to solve the model based on the Runge-Kutta method using Matlab. The vibration acceleration curve of the periodic change of the gear system was obtained, and the correctness of the dynamic model was verified. The transmission error response curve exhibits time-varying characteristics, which is consistent with the change of time-varying stiffness.

By comparing two kinds of dynamic response curves with and without return difference, this paper illustrates the influence of return difference on dynamic transmission error in a gear system. The response of the gear drive without return difference decays quickly after the load torque becomes zero. The load of the gear system with return difference decays very slowly. After the torque becomes zero, there will be multiple oscillations, and the amplitude is larger than the amplitude of the shock of the gear system without return difference.

\section{References}

1. Saxena A, Chouksey M, Parey A. Effect of mesh stiffness of healthy and cracked gear tooth on modal and frequency response characteristics of geared rotor system[J]. Mechanism and Machine Theory, 2017,107:261-273.

2. Fernandez-del-Rincon A, Garcia P, Diez-Ibarbia A, et al. Enhanced model of gear transmission dynamics for condition monitoring applications: Effects of torque, friction and bearing clearance $[\mathrm{J}]$. Mechanical Systems and Signal Processing, 2017,85:445-467.

3. Wang J, He G, Zhang J, et al. Nonlinear dynamics 
analysis of the spur gear system for railway locomotive $[\mathrm{J}]$. Mechanical Systems and Signal Processing, 2017,85:41-55.

4. Guangjian W, Lin C, Li Y, et al. Research on the dynamic transmission error of a spur gear pair with eccentricities by finite element method[J]. Mechanism and Machine Theory, 2017,109:1-13.

5. Saghafi A, Farshidianfar A. An analytical study of controlling chaotic dynamics in a spur gear system[J]. Mechanism and Machine Theory, 2016,96:179-191. 